\title{
Sizing inductors of an axial flux synchronous generator
}

\author{
D. Lahouel, D. Hedjazi, R. Abdessemed \\ Electrical Engineering Department, Mustafa Ben Boulaid University, Batna 2, Algeria
}

\begin{tabular}{l}
\hline \hline Article Info \\
\hline Article history: \\
Received Jan 19,2018 \\
Revised Jun 9,2019 \\
Accepted Jun 24, 2019 \\
\hline Keywords: \\
Axial flow generator \\
CAD \\
Coils sizing \\
Comsol multiphysics \\
Finite elements \\
Magnetic vector potential \\
Voltage
\end{tabular}

\begin{abstract}
This given the outcome of environmental situations in recent years, it has become necessary to use other resolutions for the energy production we need, and among these wind power sources. Since the advent of windmills to the first wind turbines, the technology of aero-motors (or wind sensors) has evolved rapidly. This article presents a study of an axial flow generator, and this mechanism is based on the energy of the wind turbine, this structure solves the problem of the distribution of the magnetic field lines in the armature coils and the inductor of the generator, observing the influence on the voltage from the linear velocity, the density of the injected current and the position along its axis; and that by the method of Finite Elements and is used the computer aided design (CAD), and more particularly the software comsol multiphysics to obtain the results that the good operation of the generator.
\end{abstract}

Copyright $(0) 2019$ Institute of Advanced Engineering and Science. All rights reserved.

\section{Corresponding Author:}

D. Lahouel,

Electrical Engineering Department,

Mustafa Ben Boulaid University,

Rue Chahid Boukhlouf Med El-Hadi 05000 Batna, Algeria.

Email: d_lahouel@yahoo.fr

\section{INTRODUCTION}

Day after day, there has been a significant production and consumption of electricity that has led to the greatest leakage of the earth's resources, and this is what we have experienced (the depletion of the resources of the earth, environmental pollution, global warming, climate change).

Faced with this dilemma, it is necessary to resort to new sources of energy which will be of no consequence to man and the environment. Thus, industrialized countries have embarked on the development and use of renewable energy sources such as solar, biomass, geothermal, hydro, etc.

Among these energy sources, the wind turbine represents a fairly large potential not to replace the existing energies but to compensate for the dampening of the demand more and more galloping. After centuries of evolution and more advanced research in recent decades, several countries have now turned resolutely towards wind power.

The operation of electric machines is based on the action of the electromagnetic field, which can be constant or variable over time and often in space. Knowledge of the magnetic field therefore makes it possible to have access to the calculation of the performance and operation of this device, the evaluation of this field at each point of the space which makes it possible to deduce the electromotive forces for a function in generator or torques for motor operation.

The process of designing an electrical machine goes through several stages, one of which is the evaluation of electric and magnetic fields. [1-2].

This work is devoted to the design and analysis of the electromagnetic field in an axial generator wound coil taking into account a study a synchronous generator with permanent magnets (SGPM) with axial flow of power $0.8 \mathrm{Kw}$, the operation is carried out in proceeding as follows:

- Design of the SGPM with axial flow (Comsol).

- Determination of the magnetic field B and the output voltage. 
- Sizing of the winding inductor of the axial flow generator of power $0.8 \mathrm{Kw}$, conception and analysis of the machine under Comsol.

- Comparative study between the two generators.

The computer-aided design tool CAO Comsol allows us to solve the problem of the distribution of magnetic field lines in the machine and to observe the influence of the density of the injected current and the dimensions of the inductive coils on the voltage release.

\section{MODELING APPROACH}

The study of electromagnetic phenomena in electrical machines is based on the use of Maxwell's as shown in (1), because they reflect the interactions between electrical and magnetic graders, while taking into account the reference framework associated with the medium to be studied.

$\operatorname{rot} \vec{H}=\vec{J}+\frac{\partial \vec{D}}{\partial t}$

$\operatorname{rot} \vec{E}=-\frac{\partial \vec{B}}{\partial t}$

$\operatorname{div} \vec{B}=0$

$\operatorname{div} \vec{D}=\rho$

Supplemented with specific materials:
$\vec{B}=\mu_{0} \mu_{r} \vec{H}$
$\vec{D}=\varepsilon \vec{E}$
$\vec{J}=\sigma \vec{E}$
(c)

For low frequencies $\frac{\partial \vec{D}}{\partial t}$ is zero [3-5].

In the case of electric machines, the most suitable model for the study of electromagnetic phenomena is magnetodynamics, since it takes into account the speed and the currents that are variable with respect to time.

Magnetodynamics have the following advantages:

- It is the most used since it reduces the number of unknowns;

- It makes it possible to impose electric currents, and the deduction of any other physical magnitude. Knowing that :

$\operatorname{rot} \vec{E}=-\frac{\partial \vec{B}}{\partial t}$ et $\vec{B}=\operatorname{rot} \vec{A}$

$\operatorname{rot} \vec{E}=-\frac{\partial}{\partial t}(\operatorname{rot} \vec{A})=-\operatorname{rot}\left(\frac{\partial \vec{A}}{\partial t}\right)$

$\Rightarrow \operatorname{rot}\left(\vec{E}+\frac{\partial \vec{A}}{\partial t}\right)=\overrightarrow{0}$

The parameter $\left(\vec{E}+\frac{\partial \vec{A}}{\partial t}\right)$ is a conservative field, therefore it derives from an electrical scalar potential U, such that:

$\vec{E}+\frac{\partial \vec{A}}{\partial t}=-\overrightarrow{g r a d} U \Rightarrow \vec{E}=-\left(\frac{\partial \vec{A}}{\partial t}+\overrightarrow{g r a d} U\right)$

Since the inductors are provided with a velocity $\vec{\vartheta}$ in a magnetic induction field $\vec{B}$ which gives rise to induced density currents $\vec{J}$ is, according to the law of Laplace we have: 
$\vec{J}=\sigma \vec{\vartheta} \wedge \vec{B}$

According to (3-6), the induced currents $\sigma \partial \vec{A} / \partial t$ the induced currents must be replaced by:

$\sigma \frac{\partial \vec{A}}{\partial t}+\sigma \vec{\vartheta} \wedge \vec{B}=\sigma \frac{\partial \vec{A}}{\partial t}+\sigma \vec{\vartheta} \wedge \operatorname{rot} \vec{A}$

Thus, we arrive at the general equation of magnetodynamics:

$$
\operatorname{rot}\left(\frac{1}{\mu} \operatorname{rot} \vec{A}\right)+\sigma \frac{\partial \vec{A}}{\partial t}+\sigma \overrightarrow{\operatorname{grad}} U-\sigma(\vec{\vartheta} \wedge \operatorname{rot} \vec{A})=\overrightarrow{J_{\text {ext }}}
$$

Equation (8) is of the integro-differential and non-stationary type, its resolution makes it possible to determine the vector potential $\vec{A}$, and any other parameters since every derivative of $\vec{A}$.

To solve this type of equation, generally we use the method of finite differences, or finite elements, for our case the operation of a CAD software Comsol makes it easier for us the task.

\section{RESOLUTION TOOL}

Comsol multiphysiques (FEMLAB) is a numerical simulation tool that allows to treat many problems coupled thanks to its integrated bank and of very varied physical equations, represented by partial differential equations. This software has many advantages such as:

- the use of a graphical interface

- contains most of the equations;

- can communicate with other software such as MATLAB, SolidWorks. [6-7].

The use of this software allows us a numerical simulation as close as possible to real systems. While respecting the following steps:

- Knowledge of the dimensions of the model to be studied

- Schematics of geometry

- Discretization of the domain and solving the problem

- Results in the form of a curve or text

\section{DESIGN OF THE TWO VARIANTS}

\subsection{Synchronous generator with permanent axial flux magnets (SGPM).}

The axial flow synchronous generator consists of a ferromagnetic steel disk called a rotor, on which are placed permanent magnets, allowing the production of a magnetic field. The fixed stator part carries slots, in which are put three three-phase windings, which can be operated either star or triangle [8-9].

In this part of our work, we will try to design a SGPM with power $0.8 \mathrm{~kW}$, characteristics (see Table 1), and nameplate.

Table 1. Characteristics of the SGPM

\begin{tabular}{cc}
\hline Part & Characteristics \\
\hline Nominal voltage & $150 \mathrm{~V}$ \\
Nominal rotation speed & $1500 \mathrm{~min}-1$ \\
Nominal power & $0,8 \mathrm{~kW}$ \\
Stator : Wired & 24 Slots \\
& 4 poles \\
Rotor: Permanent magnets & $8(55 \times 35 \times 8), \mathrm{Br}=0.84 \mathrm{~T}$ \\
w0 = $(11+12+13)(\mathrm{mm})$ & 60 \\
R0 $-\mathrm{R} 1(\mathrm{~mm})$ & $55-140$ \\
\hline
\end{tabular}

On the basis of data of the generator, one moves to the Comsol software to schematize the SGPM (Figure 1), and simulates the system. Figure $1(\mathrm{a}, \mathrm{b})$ represents the front view in 3D, and the left view of the generator. 


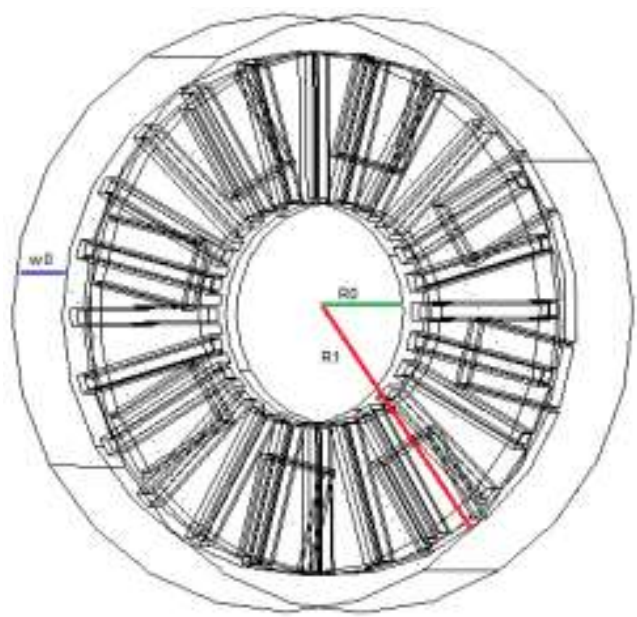

(a)

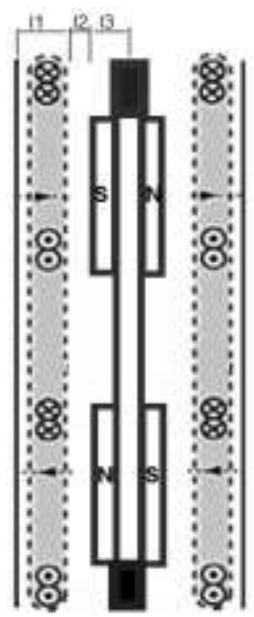

(b)

Figure 1. Geometry of the axial flow synchronous generator

To determine the various parameters of the SGPM, one must ensure that the design by CAD does not contain an error, and then one moves to the discretization and the resolution of the problem. The results obtained are shown in Figure 2.

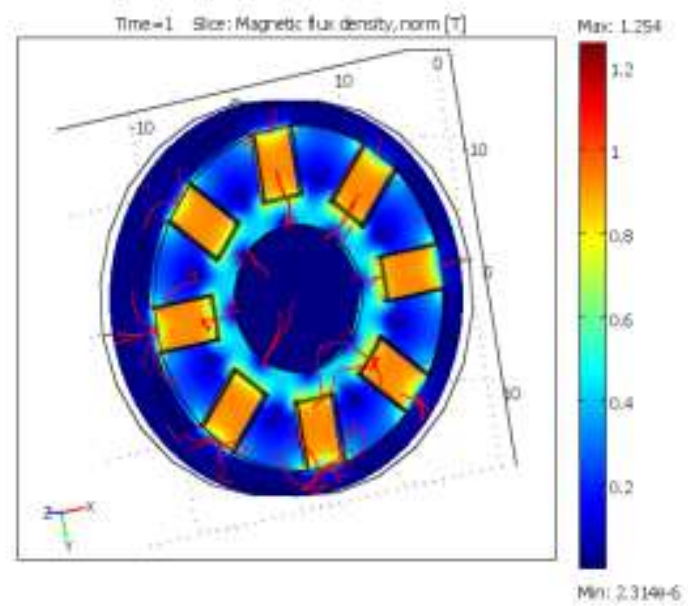

Figure 2. Distribution of SGPM magnetic field lines B in 3D

Figure 3 shows the variation of the magnetic induction at the air gap in the dynamic state (rotor equipped with a rotational speed), the maximum value of $\mathrm{B}$ is of the order of $0.93 \mathrm{~T}$. This induction gives rise to a f.e.m in the stator windings. Figure 4 shows the value of the output voltage, measured at the terminals of a single stator coil, with an effective value of $50 \mathrm{~V}$. 


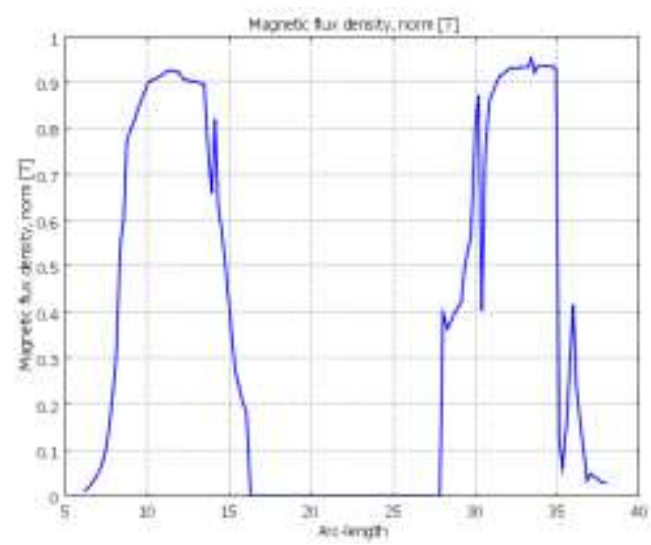

Figure 3. Magnetic induction $\mathrm{B}$ as a function of the angle of rotation

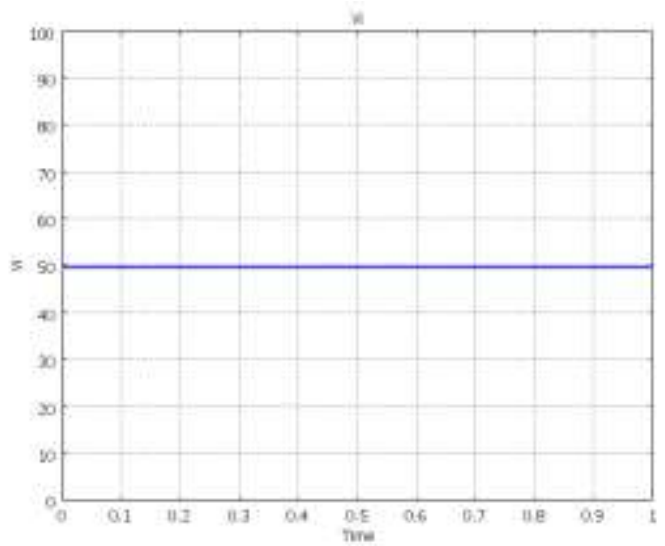

Figure 4. The output voltage at the terminal of a stator coil

Our discoid generator consists of two symmetrical parts, and the results correspond to only one stator side. The voltage obtained for $\mathrm{B}=0.93 \mathrm{~T}$ at the air gap clearly reflects the value indicated on the rating plate. The aim of this work is to dimension and replace the permanent magnet inductor by a coiled inductor, while keeping the same stator.

\subsection{Axial flux generator with wound inductor}

The inductor in question is discoid, on which are mounted eight coils (Figure 5), capable of producing the same magnetic induction in the air gap of the order of 0.93T. The coils are of circular shape (Figure 6).

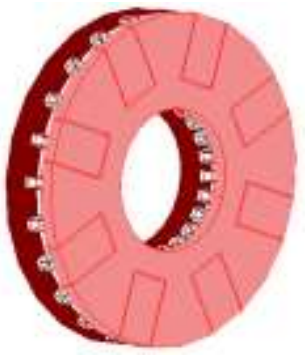

(a). Wound stator of generator

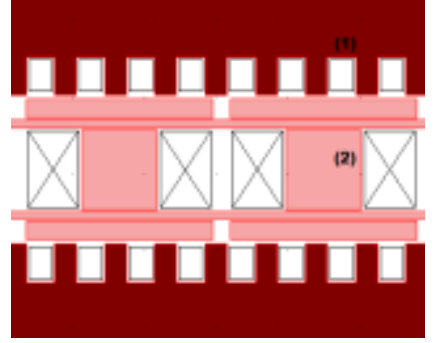

(b). Wound inductor of generator

Figure 5. Geometry of the wound inductor with axial flux 


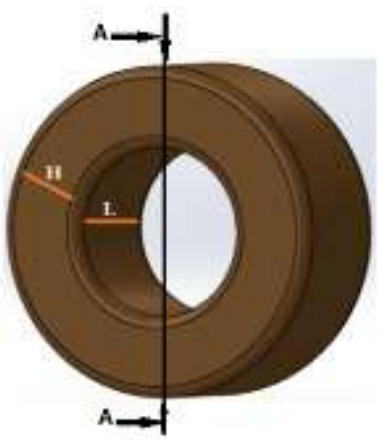

(a)

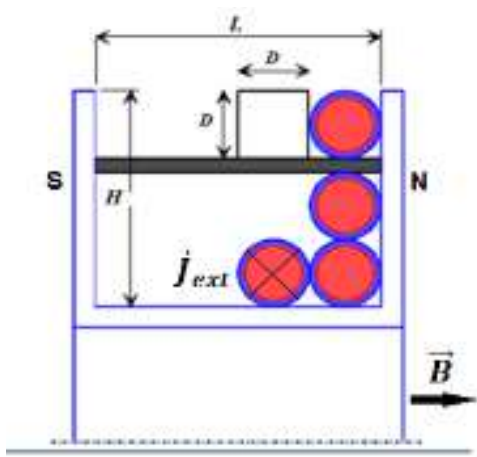

(b)

Figure $6(\mathrm{a}, \mathrm{b})$ Front view and section of a coil's inductor

Determination of dimensions is of great interest to the design of electrical machines. Indeed, the dimensioning and evaluation of a coil of the axial generator relies on the knowledge of the magnitudes of the electromagnetic fields, and among these magnitudes the induction of the magnetic field of value practically between 1.4 and $1.6 \mathrm{~T}$ and a density of current $\mathrm{J}$ which may be between 5 and $7 \mathrm{~A} / \mathrm{mm}^{2}$.

The parameters are determined as follows:

$\mathrm{S}_{\mathrm{b}}$ (la surface bobinée $)=(\mathrm{H} \times \mathrm{L})(\mathrm{mm})$

$\mathrm{J}_{\mathrm{ext}}=6 \mathrm{~A} / \mathrm{mm}^{2}$

For winding

From this data, it is possible to add: the cross-section of the conductive element, the current that can be injected into this surface, and the number of turns of the coil. The following table gives the diameters of the winding wire and the corresponding currents:

Table 2. Conductor Diameters as a Function of $\mathbf{J}$ and Corresponding Currents

\begin{tabular}{cccc}
\hline Twire $\mathrm{D}_{\mathrm{c}} / \mathrm{mm}$ & $\begin{array}{c}\text { Current } 1 \\
\mathrm{~J}=5 \mathrm{~A} / \mathrm{mm}^{2}\end{array}$ & $\begin{array}{c}\text { Current 1 } \\
\mathrm{J}=6 \mathrm{~A} / \mathrm{mm}^{2}\end{array}$ & $\begin{array}{c}\text { Current } 1 \\
\mathrm{~J}=7 \mathrm{~A} / \mathrm{mm}^{2}\end{array}$ \\
0.5 & 0.89 & 1.176 & 1.372 \\
0.7 & 1.925 & 2.31 & 2.695 \\
0.9 & 3.18 & 3.816 & 4.452 \\
1 & 3.925 & 4.71 & 5495 \\
1.5 & 8.835 & 10.602 & 12.369 \\
2 & 15.7 & 18.84 & 21.38 \\
\hline
\end{tabular}

Avec : $J_{\text {ext }}=I / S_{c}$

et : $S_{c}=\frac{\pi \cdot D_{C}^{2}}{4}$

Donc : $S_{b}=H \cdot L=\left(N_{c} \cdot S_{c}\right) \cdot K_{r e m p}$

$K_{\text {remp }}$ : The coefficient of filling

We suppose : $K_{\text {remp }}=1$

et : $\mathrm{J}_{\mathrm{ext}}=6 \mathrm{~A} / \mathrm{mm}^{2}$

$I:$ the intensity of current to be injected

$\mathrm{S}_{\mathrm{c}}$ : conductive element surface

$\mathrm{N}_{\mathrm{c}}$ : the number of turns

$\mathrm{S}_{\mathrm{b}}$ : surface wound, AN: $S_{b}=(14 \times 26)\left(\mathrm{mm}^{2}\right)$

We suppose : $K_{\text {remp }}=1$ 
Then, from these dimensions, and in addition the dimensions of the permanent magnet of the first model, the schematization of the inductor element is as follows:
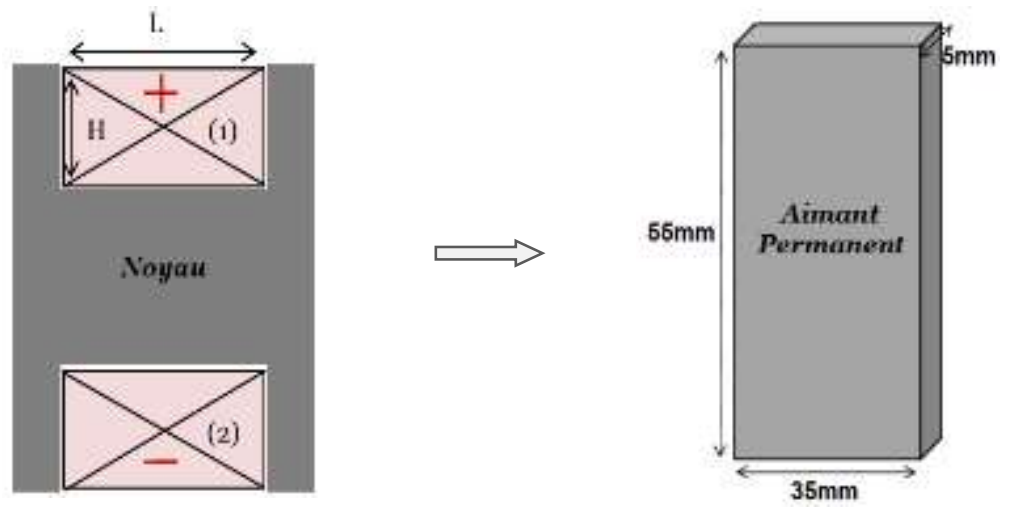

Figure 7. Permanent magnet and inductive coils

After the dimensioning of a coil, the permanent magnets of the coils are replaced, while maintaining the same armature. While respecting the design steps of the Permanent Magnet variant, we must do the same work. Viewing the time of resolution which is important, we try to take a part of the machine, according to the parameters of the Table 3. The characteristics of the generator in question are shown in Table 3 which summarizes the set of electrical and geometric dimensions.

Table 3. Generator Geometric Dimensions

\begin{tabular}{|c|c|c|c|}
\hline Elements & Dimensions & Symbols & Values $(\mathrm{mm})$ \\
\hline & Lengths & $\mathrm{L}$ & 175 \\
\hline & Width & 1 & 30 \\
\hline \multirow[t]{3}{*}{ Induced } & Height & $\mathrm{h}$ & 70 \\
\hline & Number of Slot & $\mathrm{m}$ & 12 \\
\hline & Length & $\mathrm{L}_{0}$ & 26 \\
\hline Inductor & Diameter & $\mathrm{D}_{0}$ & 14 \\
\hline \multirow{3}{*}{ (4 coils) } & Length & $\mathrm{L}_{1}$ & 37 \\
\hline & Diameter & D & 12 \\
\hline & Diameter of conductor & $\mathrm{d}$ & $0.3-0.4$ \\
\hline Gap & Thickness & $\delta$ & 3.4 \\
\hline
\end{tabular}

Schematization and solving the problem performed under Comsol.

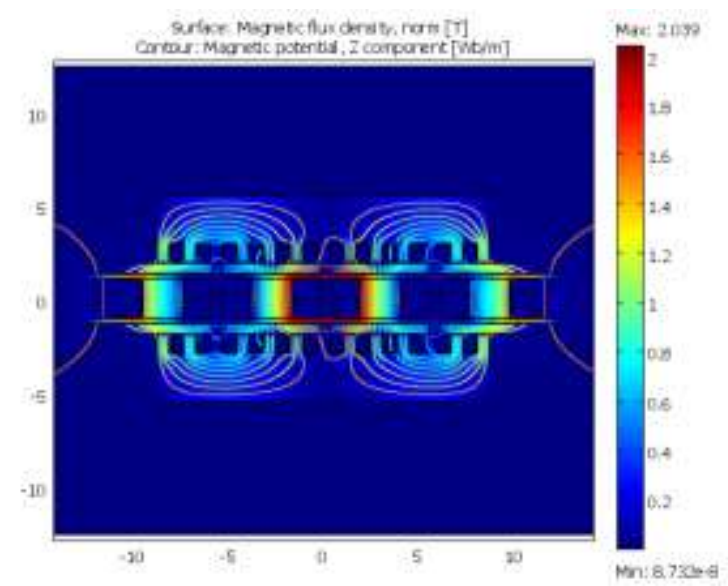

Figure 8. Distribution of magnetic field lines in 2D 
Figure 9 shows B at the air gap under dynamic conditions at the maximum values of $1.4 \mathrm{~T}$ (pole in front of the tooth) and $\mathrm{B}=1.1 \mathrm{~T}$ (pole opposite the slot).

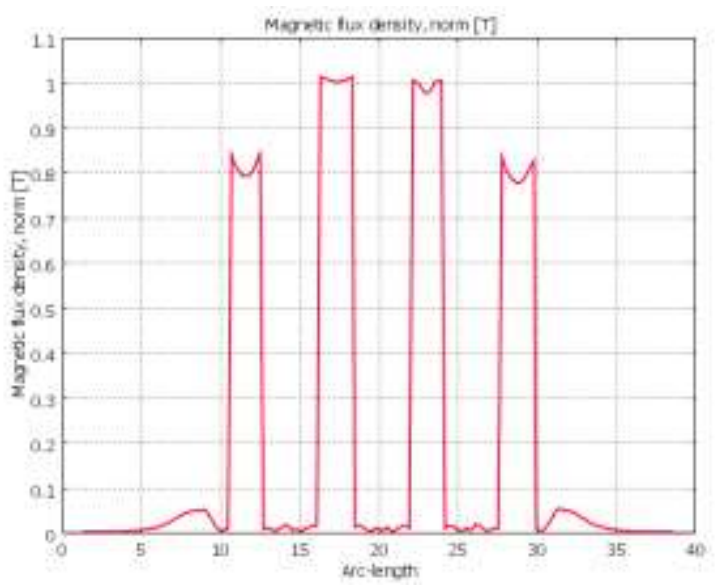

Figure 9. Magnetic induction at the air gap under dynamic conditions

The output voltage at the terminals of the armature coil is of the order of $50 \mathrm{~V}$.

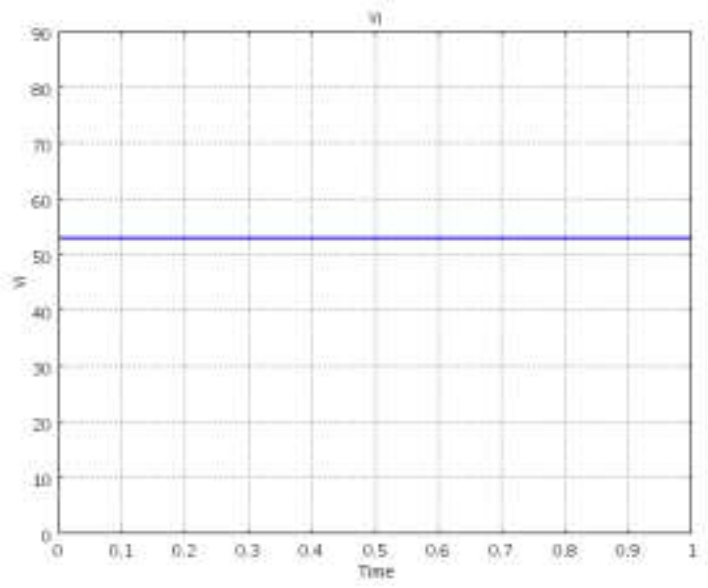

Figure 10. The resulting voltage in the armature windings

In addition to the previous electromagnetic study, the thermal effect can be deduced for the two models, and the most suitable generator between the permanent magnet inductor model and the coil inductor.

\section{THERMAL STUDY}

This study is carried out for both models ( $\underline{S G P M}$ and SGIB), for a duration of $1800 \mathrm{~s}$, and the results are given in the form of Figures 11 ( $\mathrm{a}$ and $\mathrm{b}$ ).

It is noted that the mean temperature at the air gap of the $S G P M$ has evolved from $293 \mathrm{~K}$ to $390 \mathrm{~K}$ during the operating time of the machine with a current density of $3 \mathrm{~A} / \mathrm{mm}^{2}$ (Figure $11 \mathrm{a}$ ). On the other hand, for the GSIB working under the same conditions and configurations, the temperature evolves from $293 \mathrm{~K}$ to $360 \mathrm{~K}$ (Figure $11 \mathrm{~b}$ ) 


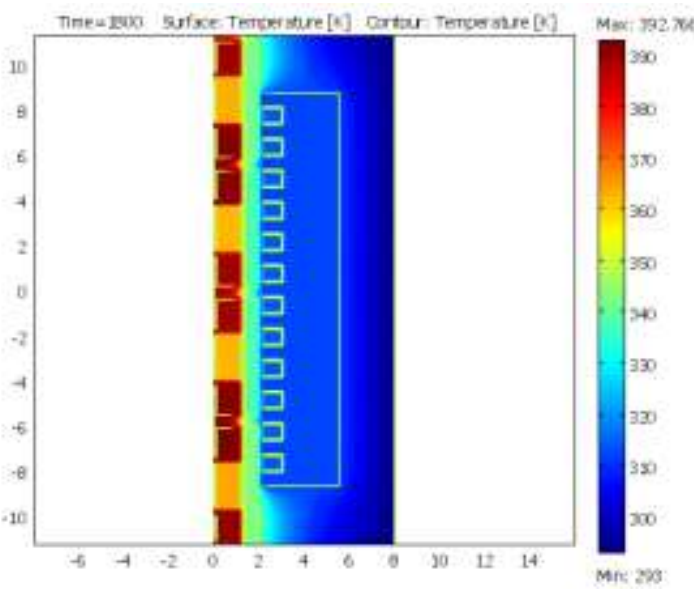

(a)

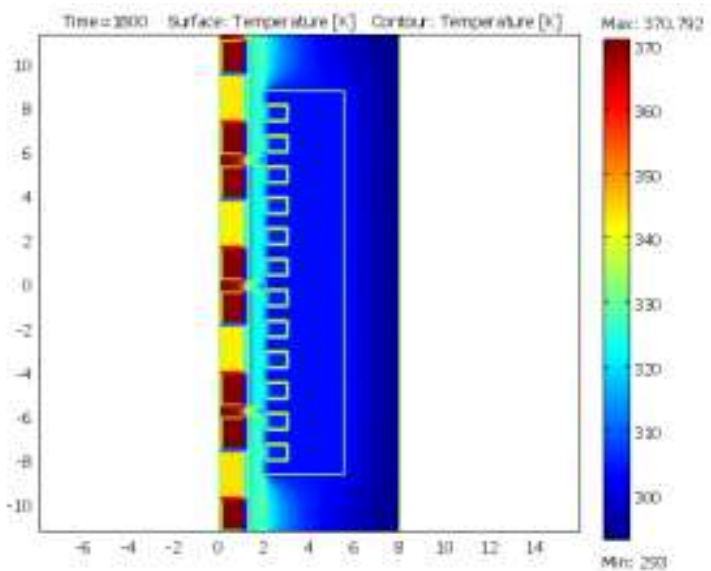

(b)

Figure 11. Temperature distribution in SGPM and SGIB after $1800 \mathrm{~s}$

The temperature field is almost uniform for both cases, and with the same initial conditions (dimensions, current density, ambient temperature, etc.), the results of the coil inductor are better compared with the permanent magnet generator. The results of the temperature as a function of time for a duration of $1800 \mathrm{~s}$, were recorded along the air gap of the two models, are shown in Figure 12 (a, b).

Figure 12 (a) shows the variation of the temperature at the air gap of the GSAP whose max value is of the order of $345 \mathrm{~K}$. Figure 12 (b) shows the variation of the temperature at the air gap of the GSIB whose max value is of the order of $325 \mathrm{~K}$. For a time greater than $1800 \mathrm{~s}$, the temperature difference increases which requires a cooling circuit for both models.

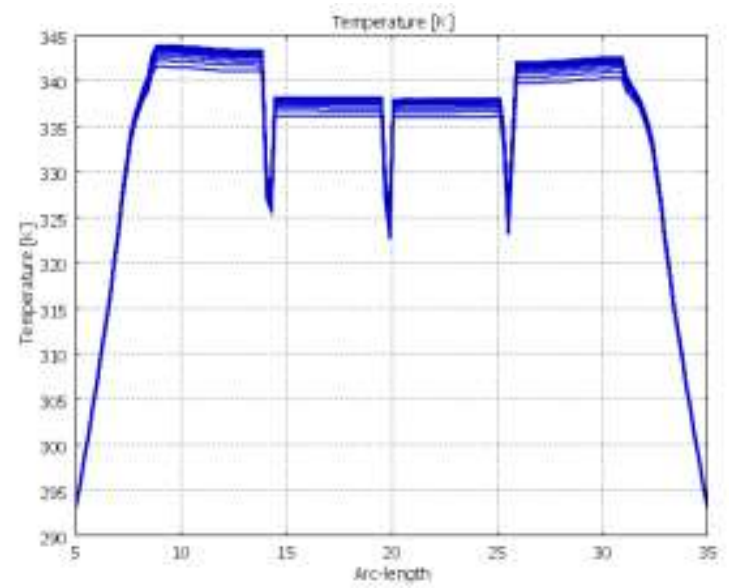

(a)

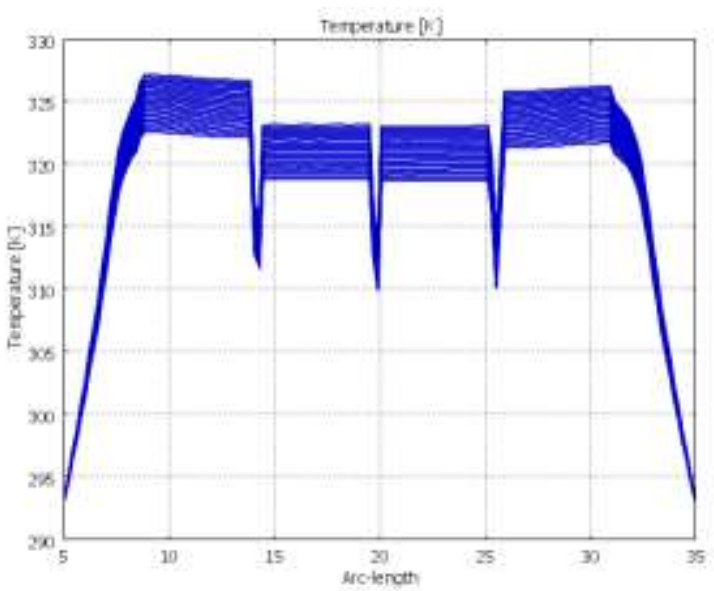

(b)

Figure 12. The temperature profile in the air gap for the two models successively (GSAP and GSIB)

Throughout the analysis, the results obtained for the case of two machines are very close, such as the magnetic induction at the air gap, and the output voltage of two generators, as well as the temperature. The replacement of two magnets (face to face), by a single coil, allowed us during this study to:

- Adjust the magnetic induction and the output voltage while acting on the excitation current.

- Decrease the thermal effect

The major disadvantage of the GSIB is the necessity of the brush rings at the level of the inductor. The experiment carried out by CAD makes it easier for us to calculate and dimension a coil equivalent to a given permanent magnet. 


\section{CONCLUSION}

The transformation of the permanent magnet inductor by a wound inductor is not an easy task, even through CAD tools, since these tools require a great deal of control. In our case, and in order to try to facilitate the work, the inductor has been dissected to finally dimension a coil while taking into account the induction in the air gap of the axial flux generator and the number of poles. On the basis of these parameters, an attempt has been made to dimension a single coil corresponding to the permanent magnet of the reference variant. The rewinding of the coil inductor in place of the permanent magnet, allowed us to analyze and recover the results such as induction and output voltage, to make a comparison between the two machines. From the results obtained, it was observed that they are very close even if these two machines do not keep the same weight and volume.

\section{REFERENCES}

[1] H. Allag, M. E. H. Latreche, M. R. Mekideche, N. Ikhlef., "Axial Magnetic Forces Comparison in Linear and Nonlinear Model of Ferromagnetic Core of Magnetic Actuators," Medwell Journals, vol. 7, no. 2, pp. 1199-1202, 2007.

[2] R. Blanchet, F. Gaume et A. Bondeau, "Optimal Distribution of Current in an Iron-Free Coil for Measuring of Magnetic Susceptibility," Revue of apply Physics Mars, 1973, vol. 83.

[3] Bennecib Nedjoua, "Contribution to the Study of a Conductive MHD Machine for Electrical Network Operation," University of Batna. Algeria. 2010.

[4] Hedjazi Djemai, "Study and Design of a Double Induction Linear Motor," University of Batna. Algeria. 1994

[5] J. Faiz, M. Ebrahimi-Salari and G. Shahgholian, "Reduction of Cogging Force in Linear Permanent-Magnet Generators," in IEEE Transactions on Magnetics, vol. 46, no. 1, pp. 135-140, Jan. 2010.

[6] André Buchau, Wolfgang M. Rucker, "Analysis of a Three-phase Transformer Using COMSOL Multiphysics and a Virtual Reality Environment," Universität Stuttgart, Stuttgart, Germany, 2011.

[7] Dudnikov Evgeny, Shmelev Vjacheslav, Walde Bertil, "Educational electronic course "Theory of the Electromagnetic Field" on the Basis of the Program Complex COMSOL Multiphysics. Institute of Control Problem," Moscow, Russia, Vladimir State Technical University, Vladimir, Russia.

[8] Garrison F. Price, Todd D. Batzel, Mihai Comanescu, Bruce A. Muller, "Design and Testing of a Permanent Magnet Axial Flux Wind Power Generator," Pennsylvania State University, Altoona College.

[9] Building stacked up axial alternators, Scoraig Wind Electric. UK. 